\title{
3D Visualization of Nanoscale Tomography Using Holographic Displays
}

Jacob Pietryga and Robert Hovden

University of Michigan, Ann Arbor, Michigan, United States

Perspective painting was a revolutionary tool which enabled polymaths of art and engineering to understand the 3D world around them - fueling the scientific revolution that would shortly follow. Now, modern science is being transformed through a digital revolution. However, the ability to express 3D datasets is dimensionally restricted to a $2 \mathrm{D}$ canvas or digital display. This is particularly true in the threedimensional characterization of materials at the nano- and meso-scale, whose importance has extended to a wide class of nanomaterials that will define the future of clean energy, medical imaging, and computing. The deluge of digital data acquired by the modern scientist requires new methods for visualization to rapidly discern 3D morphology and symmetry. Augmented, mixed, and virtual reality offer new routes to understanding 3D datasets and new possibilities for electron tomography.

Here we develop 3D holographic visualization of nanoscale material datasets obtained from scanning transmission electron microscope (STEM) tomography. Holographic displays bring extended reality into a shared experience for all scientists in a room-without the use of 3D glasses or synchronized virtual reality headsets. This nascent technology fits natively alongside an electron microscope workspace to enhance 3D characterization and scientific collaboration (Fig. 1).

Autostereoscopic multiview displays based on lenticular arrays are the most convincing holographic display available (Fig. 2d). Cylindrical lenses known as "lenticules" diffract light from a set of pixels to a range of angles that generate the view cone (Fig. 2c) [1]. Unique views are defined in angular increments throughout this view cone and receive a fraction of the display's total pixels. For example, the current large LookingGlass holographic display[2] distributes 3840 × 2160 pixels across 45 views in a $\sim 45^{\circ}$ view cone - this translates to approximately 572 x 322 pixels per view in $\sim 1^{\circ}$ increments (Fig. 2a). The large number of unique viewing angles simulates an object lightfield, introducing subtle binocular cues that efficiently convey depth information to all observers [3].

The LookingGlass holographic display has been adapted and integrated with nanoscale tomography datasets using our in-house developed tomography tool, tomviz. After a tomographic reconstruction is complete, 3D tomograms are rendered from multiple angles - scale cubes, axes, and lighting can be added to enhance depth information and contrast in the tomographic model (Fig. 2b). The angle-dependent tomographic renderings are distributed across pixels under the lenticular array such that they diffract at the angle they were rendered (Fig. 2c). An observer will see views that correspond to their viewing angle, and different images seen by each eye invoke binocular visual cues [1]. As a result, the multiview display outputs a convincing 3D tomographic model that feels palpable (Fig. 2d) [4]. The holographic display can accommodate volumetric and contour models, and integration of haptic tools such as Leap Motion enable intuitive 3D manipulation of these datasets.

$3 \mathrm{D}$ visualizations are informationally dense representations of nanomaterials, but interpretation is limited by $2 \mathrm{D}$ mediums that lack the natural depth cues provided by binocular vision and multiple viewing angles. With holographic displays, the interpretation of 3D nanoscale materials feels intuitive and familiar while also allowing simultaneous interaction with multiple colleagues [6]. 


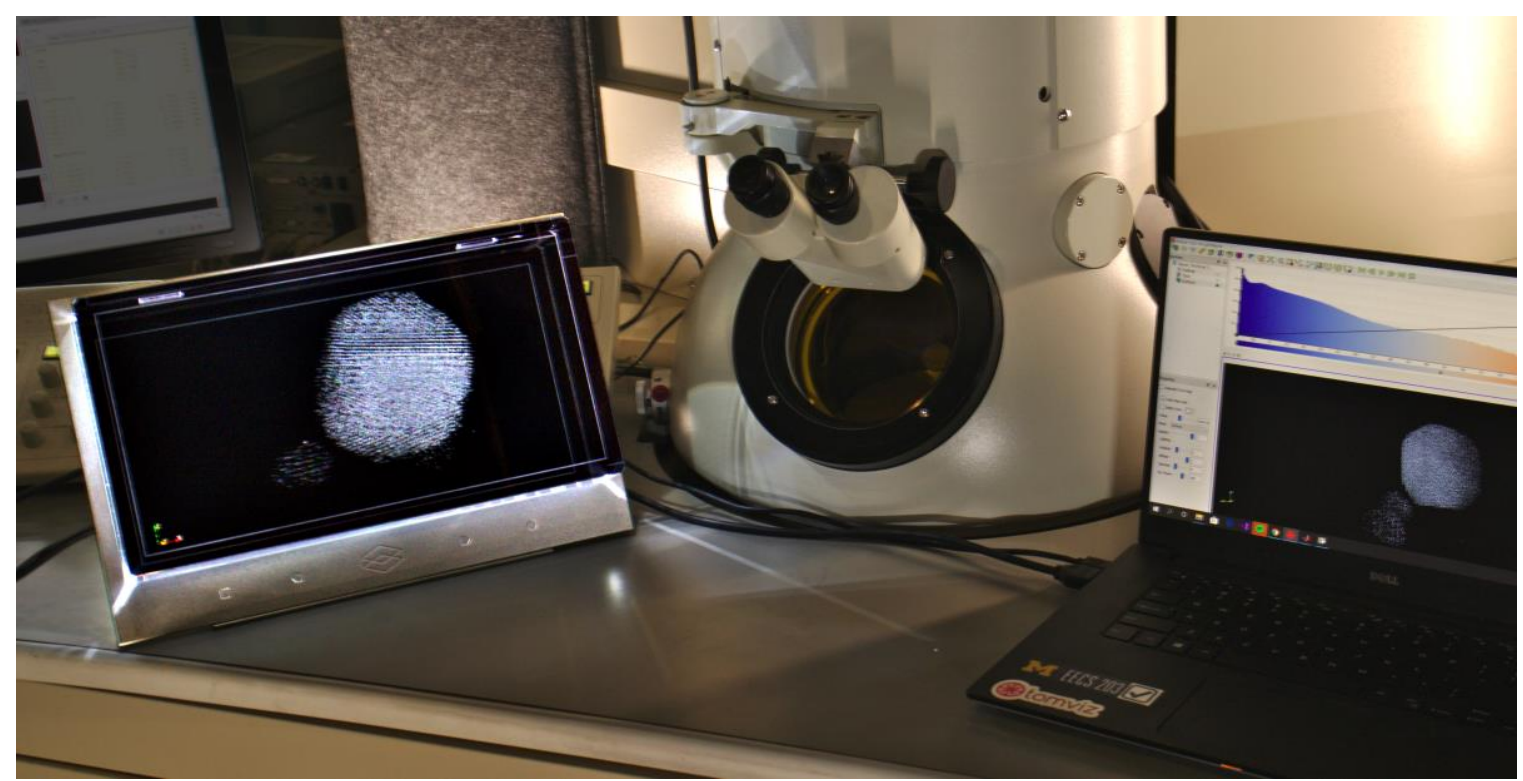

Figure 1. Electron tomography environment with LookingGlass holographic display. A transmission electron microscope (TEM) is used to collect projection images of nanomaterials across a wide range of angles. Tomographic software reconstructs this data and then translates it to a holographic display.
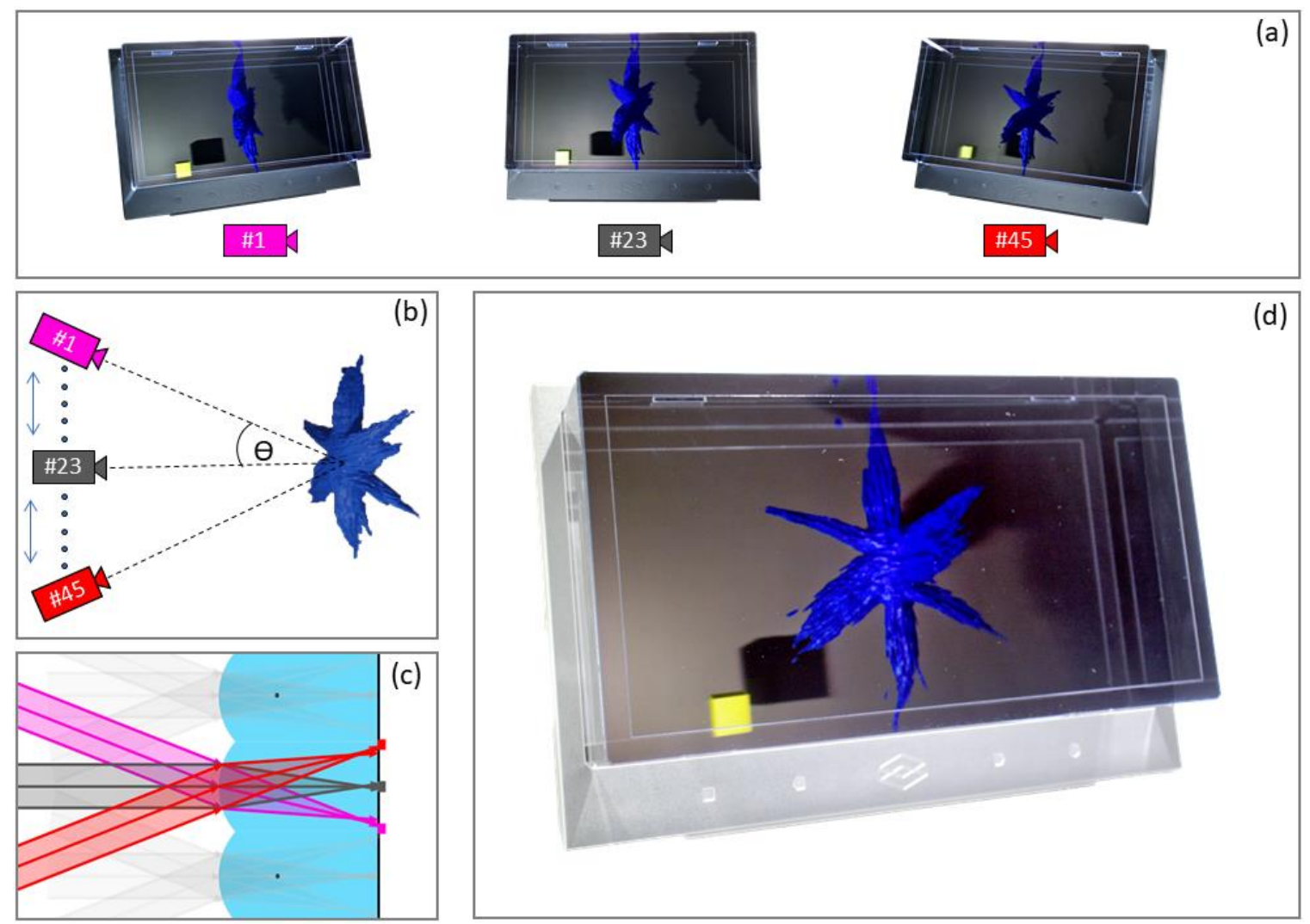

Figure 2. STEM tomography of hyperbranched Co2P particle pipeline on holographic display. (a) Visual representation of the multiview display observed from extreme angles. Depth information is conveyed through lighting and shadows as well as relative position and size. (b) Usage of holographic displays begins with generating 45 unique views focused on a point on the sample. The maximum viewing semiangle $\Theta$ is $\sim 22^{\circ}$ for best viewing experience. (c) A single lenticule's ray diagram is shown with basic pixel 
mapping. Adjacent pixels smooth transition between neighboring views. (d) Final render of Co2P displayed on LookingGlass holographic display with lighting and $50 \mathrm{~nm}$ scale cube. To a viewer, binocular cues make the nanomaterial appear 3D.

\section{References}

[1] Holliman, N. (2002). 3D Display Systems. 38.

[2] LookingGlass Factory (2020). Looking Glass 15.6" Development Kit \& 15.6" Pro.

[3] McKee, S. P., \& Taylor, D. G. (2010). The precision of binocular and monocular depth judgments in natural settings. Journal of Vision, 10(10):5, 1-13.

[4] Pang, B., et al. (2017). High-efficient rendering of the multi-view image for the three-dimensional display based on the backward ray-tracing technique. Optics Communications, Volume 405, 306-311.

[5] Levin, B., et al. (2016). Nanomaterial datasets to advance tomography in scanning transmission electron microscopy. Scientific Data. 3. 160041.

[6] Authors acknowledge support from The University of Michigan's XR Clinic and Academic Innovation in Education. 